\title{
Preface to the special issue on nanopores in bioanalytical sciences
}

\author{
Yi-Tao Long ${ }^{1} \cdot$ Tudor Luchian $^{2} \cdot \mathrm{Li}-\mathrm{Qun} \mathrm{Gu}^{3}$
}

Received: 3 January 2016 / Accepted: 5 January 2016 /Published online: 29 January 2016

(C) Springer-Verlag Wien 2016

Tremendous progress has been made in the field of nanopore technology in general, and in respective biosensing schemes. This Special Issue of Microchimica Acta contains a collection of representative articles in this field. The three Guest Editors are convinced that this issue very well reflects the rapid progress that has been made in this field and demonstrates the advanced level of nanopore technology.

Nanopore technology is likely to have a large impact on bioanalytical methods. Here, the focus is on new materials and methods, and on applications to analytical challenges. A most thrilling feature of bioanalytical nanopores technology relies on the fact that its methods will lead to new techniques rather than replacing existing ones that often perform adequately

Yi-Tao Long

ytlong@ecust.edu.cn

Tudor Luchian

luchian@uaic.ro

Li-Qun Gu

gul@missouri.edu

1 Key Laboratory for Advanced Materials \& Department of Chemistry, East China University of Science and Technology, Shanghai 200237, China

2 Department of Physics, Alexandru I. Cuza University, 700506 Iasi, Romania

3 Department of Biological Engineering and Dalton Cardiovascular Research Center, University of Missouri, Columbia, MO 65211, USA well. Nanopore technology also relies on the use of new materials, and this also requires new methods for their characterization because conventional methods (such as mass spectrometry or NMR) often are not applicable or adequate. It is becoming more and more obvious that nanopores technology is becoming a field of its own that is highly diversified which is also indicated by the fact that respective papers are published in highly diversified journals.

Bioanalytical nanopore technology is a subdiscipline of nanotechnology that has many (and additional) features. One relates to new materials and microstructures such as glass conical nanopore channels, gold nanocorals, various other nanopores and methods for their (surface) modification, methods for their preparation (by using, for example, electron beam etching or atomic layer deposition), or membranecovered nanopore chips. Another feature relates to studies on the binding of proteins to surfaces, analysis and sequencing of nucleic acids and proteins, studies on DNA translocation velocity or conformational changes of ion-binding aptamers. In terms of sensing, electrochemical methods are predominant, examples being the determination of mercury(II) or of short ss-DNA and ds-DNA as voltammetry. Three reviews (on nanopore-based DNA single molecule detection and sequencing, on nanopore-based nucleic acid analysis, and on the detection of various biochemical species) complete the issue.

We would like to take this opportunity to thank all the authors for their tremendous efforts in preparing such excellent articles. We also thank the many reviewers that have made numerous constructive comments on the manuscripts and thereby have further contributed to the quality of the papers. 